\section{Transcranial Photobiomodulation in The Treatment of Major Depression}

\section{Abstract}

The use of phototherapy has become popular and associated with this panorama, we observe the growth of new techniques and uses. Among the most avant-garde we can find transcranial therapies and their use in fields such as psychiatry and neurology, treating in a therapeutic way pathologies such as depression, Alzheimer's, phobias and neurodegenerative diseases. The following article aimed to evaluate all studies related to the topic Transcranial Photobiomodulation in the Treatment of Major Depression, indexed in the base date PubMed. The studies were selected using the descriptors; depression; transcranial; photobiomodulation; infrared light and laser therapy, being limited to articles that used some type of control group within the publication period of 10 years. At the end of the review, the scarce number of articles on the topic was observed, although the results seem promising in the great majority. The following review may also raise data for future discussions on methodological issues involved, such as the lack of studies with large groups as well as the difficulty of performing double-blind studies of enormous scientific importance.

Keywords: Phototherapy; Transcranial; Depression

Received: May 08, 2018; Accepted: July 16, 2018; Published: July 20, 2018

\section{Marcelo de Oliveira Fonseca ${ }^{1}$, Cristina Pacheco Soares ${ }^{1}$, Rui Mateus Joaquim²* and André Barciela Veras ${ }^{2}$}

1 Institute of Research and Development, University of Vale do Paraíba, UNIVAP, Brazil

2 Catholic University Dom Bosco - UCDB, Neuroscience and Behavior Analysis Center, Brazil

\section{Corresponding author:}

Rui Mateus Joaquim

”ruimateus@hotmail.com

Catholic University Dom Bosco-UCDB, Neuroscience and Behavior Analysis Center, Brazil.

Citation: Fonseca $\mathrm{MO}$, Soares $\mathrm{CP}$, Joaquim RM, Veras AB (2019) Transcranial Photobiomodulation in The Treatment of Major Depression. Clin Psychiatry Vol.5 No.1:1

\section{Introduction}

According to the authors review study Cassano et al. [1], NearInfrared Reflectance (Photobiomodulation) (NIR) can be used to treat a variety of conditions, such as complaints of pain, wounds and even headaches. Similarly, the authors report the use of the technique for welfare and aesthetic purposes. According to the authors, the clinical use of NIR applied in NIR spectroscopy dates from the mid-1980s, when it was used to monitor the brain in the neonate.

Studies such as those by Bench et al. [2] and Matsuo et al. [3] ground discussions about the possible efficacy of phototherapy in the treatment of depression based on reduced prefrontal lobe blood flow. Likewise, other possibilities have arisen, such as the performance of photobiomodulation in the treatment of depression, by mitochondrial approach, as discussed in the study by Cao et al. [4]. Thus, the present article aims to make a brief review on the subject, investigating articles that relate the photobiomodulation in the treatment of major depression, always in studies with control groups.

\section{Methodology}

The articles were selected in the PubMed database, under the keywords: depression; transcranial; photobiomodulation; infrared light and laser therapy, being limited to articles that used some type of control group published in the last 10 years.

\section{Results}

Taking into account the evaluated criteria, 7 studies were found that fit the parameters stipulated by this review. Among the articles found, a great variety of application protocols were observed, where not only factors such as wave frequency and lasers power varied, as well as the number of sessions, the treatment time and the instruments for measuring results. Among the studies that presented good delineation, we found the study by Schiffer et al. [5], which defined areas of well-described irradiation, with a power of $250 \mathrm{~mW}$, as well as stipulated a treatment time that showed good results, although it showed a reduction of effects in the longer period (4 weeks).

In a second study, researchers Disner et al. [6] conducted a study testing the effect of ABM (Attention Bias Modification), a cognitive psychotherapeutic technique used for the treatment of major depression, associated with adjuvant transient LBI. A total of 51 adults with severe depression recruited to participate in the randomized study. Each patient was assigned to receive ABM before and after transcranial LBI. Transcranial LBI, divided among 
participants in three distinct areas of pre-frontal stimulation, was repeated 2 days after the initial stimulation and all participants underwent depressive assessments at 1 and 2 weeks.

According to the authors, the results suggest that the symptoms of depression improved, in the main, those whose stimulation focused on the right region of the frontal lobe, associated with $A B M$, compared to those who received stimulation in other areas. Still according to Disner et al. [6] it was clear that the therapeutic potential of ABM for the treatment of depression was amplified with right prefrontal LBI. The study is valuable because it describes an area of the prefrontal lobe that would offer greater therapeutic effect, but also presents some methodological limitations, since it did not compare the effects of groups submitted to LBI, with and without $A B M$, which precludes the assertion that the observed benefits are solely due to LBI. Although the study may be questioned in this sense, the authors concluded the positive effect of $L B I$ on reducing depressive symptoms.

The study of Cassano et al. [7] also aimed to investigate the effect of transcranial infrared radiation among individuals with major depression. In the study, the researchers noted that there is evidence to support the utility of transcranial infrared radiation as an antidepressant could be limited. For this reason, they organized a small-scale study involving only 4 adult patients diagnosed with major depressive disorder ( 3 men, 1 woman) in order to test the therapeutic efficacy and tolerability of infrared radiation in the treatment of major depression.

In the study, all 4 patients received irradiation at $700 \mathrm{~mW} / \mathrm{cm}^{2}$ at frequency of $84 \mathrm{~J} / \mathrm{cm}^{2}$, providing a total NIR energy of $2.40 \mathrm{~kJ}$ per session. After receiving infrared radiation (NIR), the researchers observed that depressive symptoms decreased significantly, which showed a reduction in the Hamilton Depression Scale (HAM-D) with an average score of 19.8 (pre-treatment) for 13 (posttreatment).

Researchers have not yet shown any problem with tolerability as well as no adverse reactions to infrared radiation (NIR) in patients taking part in the study. Regarding the results, two of the four participants achieved complete remission of symptoms at weeks 6 and 7, respectively. It is necessary to emphasize that none of the patients had taken drugs nor even would have carried out any type of psychotherapeutic process with scientifically proven results.

Also regarding the symptomatic remission described previously in two patients, this was consistent with responses to other antidepressants. According to the study, all 4 participants received infrared radiation (NIR), but mood improvement was not noticed in the first few weeks. Previous studies have suggested that NIR administration produces faster psychological effects by acting within just 2 weeks of administration, which was not observed in the study by Cassano et al. [7].

The researchers further hypothesized that the late onset of NIR efficacy in the study may have been related to the highest irradiation $\left(700 \mathrm{~mW} / \mathrm{cm}^{2}\right)$ and creep $\left(84 \mathrm{~J} / \mathrm{cm}^{2}\right)$. They also observed that 2 weekly NIR sessions in 4 distinct sites for 3 weeks, protocol used, may be a protocol below the ideal. It is also known that high doses of NIR (high potency and energy) may be less effective than moderate doses.

Although the study cited previously present limitations, such as the small number of participants, important factors could be observed regarding the use of photobiomodulation for depression, including: the non-invasive nature of treatment, minimal and short-term side effects of administration. In addition, it has been mentioned that this therapy may be advantageous over the first line of pharmacological antidepressant interventions that have their effects related to mitochondrial function.

Another study evaluated in this review was the authors Barrett and Gonzalez-Lima [8], the first to conduct a controlled study showing the effectiveness of transcranial laser therapy for improving cognitive and emotional function in humans. The researchers observed that photobiomodulation using light at frequency within the red the near infrared range is capable of modulating the activation of neurons in animal models and cell cultures through cytochrome oxidase photon receptors. For their study, the researchers recruited 40 healthy subjects to test the effect of low-intensity laser stimulation on cognitive performance (attention and memory) and emotion.The 40 participants divided into 2 groups of 20 and randomly assigned to receive either prefrontal LBI or a control group. In the study, LBI stimulation was administered in the forehead region with a CG-5000 diode laser. The study results indicated that individuals receiving pre-frontal LBI pacing had a substantial improvement in reaction time (as evidenced by psychomotor performance of the surveillance task) - compared to those in the control group.Still according to the authors, participants who received prefrontal LBI experienced noticeably better performance on a memory task, compared to the control group. Associated with this result, the participants' emotional state was measured before the study using the PANAS (Positive and Negative Affect Scale), according to the authors the PANAS pre-study score was compared to a PANAS score collected 2 weeks post-treatment. In this evaluation, the authors observed that the mood of the participants who received LBI showed significant improvement (as evidenced by the PANAS scale) compared to those who participated in the control group. The study demonstrated that transcranial photobiomodulation was related to the simultaneous improvement of cognition and mood factors among healthy individuals.Thus, authors Barrett and Gonzalez-Lima [8] have speculated that transcranial laser stimulation may be an effective treatment for cognitive and mood disorders in healthy individuals.

Although the results of the study suggest that transcranial photobiomodulation (with LBI) is able to improve cognition and mood, the results can only be applied to healthy individuals, since individuals with neuropsychiatric disorders (eg. those with major depression) did not participate of this study, which makes it difficult to know if they would present similar results. Similarly, the study may also be criticized as to the number of participants and the short time of treatment.

Another study that may be cited as presenting the same deficiencies for the small number of participants, but with good results, is the study by Schiffer et al. [5]. The authors organized a study to determine whether infrared photobiomodulation (NIR) 
would have a positive effect (improvement) on participants' mood, Cerebral Blood Flow (CSR), and so-called Hemispheric Emotional Valence (HEV) - without undesirable side effects.

The study had a total of 10 individuals ( 5 men, 5 women) with Major Depressive Disorder (MDD) participated in the study, it is necessary to point out that 9 patients out of the 10 who participated in the study had diagnoses of anxiety disorders comorbid. The authors also describe that of the total number of participants, 7 had a history of substance abuse, and 3 were diagnosed withpost-traumatic stress disorder PTSD (Post Traumatic Stress Disorder).

In their methodology, the authors describe that participants (10) underwent transcranial infrared (NIR) stimulation for periods of 4 minutes. Stimulation was performed at the points described in EEG, left foreface (F3) and right forearm region (F4), or participants received a placebo (the same device, without light) at the same sites previously described. Soon after the stimulation was performed, the HAM-D, HAM-A, and PANAS measurements were re-evaluated and the post-stimulation scores were compared to the baseline. The same scales mentioned above were again applied after treatment at intervals of 2 weeks and 4 weeks. Possible changes in participants' cerebral blood flow were also monitored with equipment capable of measuring total hemoglobin $(\mathrm{cHb})$ in the prefrontal cortex. According to the authors, the results indicated that within 2 weeks after treatment, 6 of 10 had reductions in depressive symptomatology, as 7 of 10 participants experienced significant reductions in anxiety symptom scores evaluated by the HAM-D and HAM- A, respectively. Concerning the increase in the expected flow in the prefrontal cortex, although the spectroscopy showed an increase in regional Cerebral Blood Flow (rCBF) after photobiomodulation, the statistical analysis did not demonstrate data relevance.

In the last factor evaluated by the study, the humoral alterations evaluated by means of PANAS scale, also presented substantially improved scores when comparing participants exposed to the treatment, in comparison to the control group. In their concluding remarks, the authors reported that throughout the study no undesirable side effects were observed and concluded that infrared light photobiomodulation could be considered an effective therapy for individuals with major depression.

Another study found within the parameters of this review was that of Cassano et al. [9]. In this study, the authors performed a double-blind study with 21 participants. Patients in the study were selected using the Hamilton Depression Rating Scale, excluding patients with severe symptoms, with a higher suicide risk, as well as other symptoms and psychiatric comorbidities. Two weekly sessions of transcranial photobiomodulation were carried out for 8 weeks in the bilateral frontal and frontal regions of F3 and F4 (reference to EEG placement sites). The conclusion of the study was that transcranial photobiomulation in Near-Infrared Reflectance (NIR) demonstrated significant antidepressant properties in patients with major depression. Pointing to the technique as a new intervention for patients diagnosed with major depression, who suffer from intolerance or refractoriness to pharmacological treatment. The authors also point out that the therapy in question is different from other non-drug techniques used for the treatment of pathology, such as devices that use magnetic modulation of neurons, in addition to presenting low cost.

The last study on the subject evaluated by this review was by Henderson and Morries [10], who evaluated 39 patients treated for TBI (Traumatic Brain Injury) between March 2013 and May 2017 who presented major depression as a comorbidity. These patients presented a diagnostic score for major depressive disorder through the Hamilton scale, as well as being evaluated by the Fast Detection Symptomatology Inventory (QIDS) before and after treatment. In the study, patients received near-infrared lasers $(810 / 980 \mathrm{~nm}$ at 8-15 W), considered high power, above 1 $\mathrm{W}$, applied to forehead and temporal regions bilaterally for 9-12 min to each area. In the study's findings, the authors describe that the study may be interpreted as the first to be effective in treating depression in high-potency transcranial photobiomodulation, according to the authors, showing superior efficacy to other studies using low potency $1 \mathrm{~W}$ ). According to the authors, the data found raise the possibility that the technique is a safe, effective and rapid treatment for depression as a comorbidity in TBI and possibly in the primary disorder of major depression, although they point out that new studies with double-blind and placebo-controlled necessary to verify such conclusions.

It is necessary to point out that the present review was limited to analyzing the human studies that fit the sieve adopted, however, it is possible to find innumerous other studies with animal experimentation that without doubt are of significant importance for the design of the technique.

\section{Discussion}

According to Hamblin [11], many researchers have already considered that transcranial photobiomodulation for cerebral disorders will become one of the most important medical applications of light therapy in the coming years. This future scenario, according to the author, could be explained not only by the good results found in studies aimed at the treatment of the most diverse neurodegenerative and psychiatric pathologies by the technique, but also by the challenges encountered by the pharmaceutical industry to present new drugs with satisfactory results and with few side effects for the same conditions. Another point that according to the author will be responsible for the possible future success of the technique lies in its low cost, which could enable the treatment of patients in their own homes still with little investment.

The present review demonstrated that among the evaluated articles, transcranial photobiomodulation could be considered a promising technique for major depressive disorder. Although its preclinical and clinical studies in humans are recent and still scarce, we can see that its results have achieved considerable efficacy, in addition to proving an inexpensive technique with few side effects. In the same way, it can be observed that, from the point of view of this review, we can describe as methodological failures of this study, its low number of participants, associated to the absence of double blind methodology, a factor that significantly increases the validity of the study. 
Although there are considerations regarding the need for clearer methodologies and better-defined protocols performed by this review, its findings do not differ from the review by Salehpour and Rasta [12], who investigated the potentials of transcranial photobiomodulation therapy in the treatment of depressive disorder. They concluding that the therapy is highly reasonable and may play an important role in non-pharmaceutical neurotherapeutic strategies, but it should be the target of further clinical and preclinical studies, which help in the elaboration of more accurate protocols for optimal dosages, as well as other physical parameters. The authors also emphasized a possible use of the technique associated with other conventional methods of treatment, which could represent better results.

It is also necessary to describe, in association with the perspectives of new therapies for major depression as well as different psychiatric and neurodegenerative pathologies, it is necessary to consider other important applications of transcranial photobiomodulation. Studies using transcranial photobiomodulation, such as those used in the optogenetic technique, present enormous potential in the investigation of neurological, neurodegenerative and neuro-psychiatric pathologies and processes, mainly by providing a better understanding of the biomolecular mechanisms involved in the synaptic dynamics and in the neural circuits, as described Pierroz and Folcher [13], Christoffel et al. [14], Chung et al. [15], Sinnen et al. [16] and Micheva et al. [17].

In this way, its importance may lie not only in the development of new therapeutics for pathologies, but also in the development of a whole range of new possibilities for basic science in neurochemistry, opening the way for a better understanding of molecular factors involved in the healthy functioning of our brain, as well as in the genesis of neurodegenerative and neuropsychiatric pathologies, as we can observe in the study by Micheva et al. [17]. In the same way, studies such as mass synaptometry try to clarify with greater detail, the mechanisms by which our brain performs its synaptic communication and how it relates to the most diverse psychiatric conditions Berlin and Isacoff [18], Kandel [19] and Gajera et al. [20].

In this way, the assimilation of transcranial photobiomodulation techniques can provide important contribution to a better understanding of healthy brain functioning, as well as to the genesis of innumerable pathologies, which can later be treated with therapies that will use the same technical basis.

\section{Conclusion}

After reading the articles published in the last 10 years, within the criteria stipulated by this review, it can be affirmed that, in all the articles, positive effects of the use of photobiomodulation for the treatment of depressive pictures were evidenced. However, the results varied with respect to symptom reduction, ranging from protocol to protocol. In the same way, it was evidenced that, although all the articles started from the same premises and objectified the same neurological alterations to obtain the therapeutic ends, each study presented a different protocol. In all protocols wavelength, energy density, methods of evaluation of depressive symptoms as well as factors such as duration of exposure and duration of treatment (number of sessions) varied. This suggests that there are still no well-defined protocols for the treatment of depression by photobiomodulation and that further studies should be carried out to establish good treatment parameters, as well as good double-blind methodologies and control groups that offer validity to the studies.

This aspect should be emphasized because studies with good double-blind design and randomization are scarce. This fact may be associated with the low scientific expression of many of the studies, as well as the limited number of researches on the subject.

\section{References}

1 Cassano P, Petrie SR, Hamblin MR, Henderson TA, Losifescu DV (2016) Review of transcranial photobiomodulation for major depressive disorder: targeting brain metabolism, inflammation, oxidative stress, and neurogenesis. Neurophotonics 3:031404.

2 Bench CJ, Frackowiak RS, Dolan RJ (1995) Changes in regional cerebral blood flow on recovery from depression. Psychol Med 25: 247-261.

3 Matsuo K, Kato T, Fukuda M, Kato N (2000) Alteration of hemoglobin oxygenation in the frontal region in elderly depressed patients as measured by near-infrared spectroscopy. J Neuropsychiatry Clin Neurosci 12: 465-471.

4 Cao X, Li LP, Wang Q, Wu Q, Hu HH, et al. (2013) Astrocyte-derived ATP modulates depressive-like behaviors. Nat Med 19: 773-777.

5 Schiffer F, Johnston AL, Ravichandran C, Polcari A, Teicher MH, et al. (2009) Psychological benefits 2 and 4 weeks after a single treatment with near infrared light to the forehead: a pilot study of 10 patients with major depression and anxiety. Behav Brain Funct 5: 46.

6 Disner SG, Beevers CG, Gonzalez-Lima F (2016) Transcranial laser stimulation as neuroenhancement for attention bias modification in adults with elevated depression symptoms. Brain Stimul 9: 780-787.

7 Cassano P, Cusin C, Mischoulon D, Hamblin MR, De Taboada L (2015) Near-infrared transcranial radiation for major depressive disorder: proof of concept study. Psychiatry J 352979.

8 Barrett DW, Gonzalez-Lima F (2013) Transcranial infrared laser stimulation produces beneficial cognitive and emotional effects in humans. Neuroscience 230: 13-23.

9 Cassano P, Petrie SR, Mischoulon D, Cusin C, Katnani H, et al. (2018) Transcranial photobiomodulation for the treatment of major depressive disorder. The ELATED-2 pilot trial. Photomed Laser Surg 36: 634-646.

10 Henderson TA, Morries LD (2017) Multi-watt near-infrared phototherapy for the treatment of comorbid depression: an openlabel single-arm study. Front Psychiatry 8: 187.

11 Hamblin MR (2016) Shining light on the head: photobiomodulation for brain disorders. BBA Clin 6: 113-124.

12 Salehpour F, Rasta SH (2017) The potential of transcranial photobiomodulation therapy for treatment of major depressive disorder. Rev Neurosci 28: 441-453.

13 Pierroz V, Folcher M (2018) From Photobiolumination to Optogenerapy, Recent Advances in NIR Light Photomedicine Applications. J Mol Genet Med 2: 10.

14 Christoffel DJ, Golden SA, Walsh JJ, Guise KG, Heshmati M, et al. 
(2015) Excitatory transmission at thalamo-striatal synapses mediates susceptibility to social stress. Nat Neurosci 18: 962.

15 Chung WS, Verghese PB, Chakraborty C, Joung J, Hyman BT, et al. (2016) Novel allele-dependent role for APOE in controlling the rate of synapse pruning by astrocytes. Proc Natl Acad Sci 113: 1018610191.

16 Sinnen $B L$, Bowen $A B$, Forte JS, Hiester BG, Crosby $K C$, et al. (2017) Optogenetic control of synaptic composition and function. Neuron 93: 646-660.

17 Micheva KD, Busse B, Weiler NC, O'Rourke N, Smith SJ (2010) Single- synapse analysis of a diverse synapse population: proteomic imaging methods and markers. Neuron 68: 639-653.

18 Berlin S, Isacoff EY (2017) Synapses in the spotlight with synthetic optogenetics. EMBO Rep 18: 677-692.

19 Kandel ER (2001) Psychotherapy and the single synapse: the impact of psychiatric thought on neurobiological research. J Neuropsychiatry Clin Neurosci 13: 290-300.

20 Gajera CR, Fernandez R, Postupna N, Montine KS, Fox EJ (2019) Mass synaptometry: High-dimensional multi parametric assay for single synapses. J Neurosci Methods 312: 73-83. 